\title{
Wiederkehrende Buchungen
}

\section{Buchhaltungs-ABC. Vieles im Alltag einer Praxis besteht aus Rou- tine - Vorgängen also, die sich in regelmäßigen oder unregelmäßi- gen Abständen wiederholen. Diese Vorgänge werden im Qualitätsmanagement der Praxis dokumentiert. Doch auch in der Buchhaltung gibt es wiederkehrende Vorfälle, und man fragt sich, ob diese jedes Mal neu identifiziert und erfasst werden müssen.}

AUTOR: BARBARA MERTENS

Wer bei seiner Buchhaltungssoftware die manuelle Eingabefunktion verwendet, muss jede Zahlung einzeln erfassen und verbuchen. Wer jedoch die Importfunktionen aus dem Online-Banking nutzen kann, hat in der Regel die Möglichkeit, auf Automatismen zurückzugreifen und spart schnell und erheblich Zeit.

Intelligente Finanzbuchhaltungssoftware bietet dem Anwender die Option, durch die Anlage von Importregeln wiederkehrende Zahlungsvorgänge für die Software erkennbar zu machen und jeder Zahlung automatisch das richtige Buchungskonto zuzuweisen. Hierfür werden herstellerabhängig unterschiedliche Methoden angeboten.

In der auf Zahnarztpraxen spezialisierten Buchhaltungs-Software fibu-doc werden beim Import von Bankdaten über die Banking-Schnittstellen die individuell anzulegenden Importregeln als Filter genutzt. Die Zuordnung ist textbasiert, weshalb die Importregeln unverwechselbar und eindeutig angelegt werden sollen. Dabei gilt: Je kürzer die Regel, desto besser für das System. Unabhängig davon, ob es sich um Einnahmen oder Ausgaben handelt, kann das Programm Zahlungen unterschiedlichen Konten zuweisen, denn fibu-doc erkennt, ob es sich um Ausgaben oder Einnahmen handelt, und ordnet die entsprechende Verbuchungsregel zu. Besonders praktisch ist es, wenn sich die Importregeln direkt aus der Erfassung einer Buchung heraus erstellen lassen. Damit ist es möglich und auch

\section{SEMINARE}

Das 1x1 der Praxiskennzahlen Mit gesundem Basiswissen sicher durch die Betriebswirtschaft 11.10.2017, München

Größer - Schneller - Besser? Mit gesundem Wachstum zum nachhaltigen Erfolg

20.10.2017, Köln
Praxisbuchhaltung:

Leicht und verständlich

(Theorieseminar)

17.11.2017, Bonn

Das 1x1 der Praxiskennzahlen Mit gesundem Basiswissen sicher durch die Betriebswirtschaft 06.12.2017, Hamburg

\section{Der FVDZ unterstüzt}

$\triangle$ Mehr Infos unter www.fibu-doc.de. Anwender von fibu-doc erhalten Support über die Hotline.

$\triangle$ Das in den Artikeln dargestellte Buchhaltungskonzept basiert auf der zahnarztspezifischen Buchhaltungssoftware fibu-doc und wird vom FVDZ unterstützt. wahrscheinlich, dass der Anwender eine Regel anlegt, sobald aus dem Buchungstext für ihn erkennbar ist, dass eine Wiederholung des Buchungspostens in Zukunft zu erwarten ist.

Dabei ist eine regelmäßige Kontrolle ratsam, ob die angelegten Regeln greifen oder geändert werden müssen. Es kann sowohl in der Praxis zu Veränderungen kommen, als auch vorkommen, dass Buchungstexte im Bankkonto sich ändern, weil der Auftraggeber diese aktualisiert. Ein Beispiel hierfür ist die Umstellung von „KZV“ auf „Kassenzahnärztliche Vereinigung“im Buchungstext der Banken, die aktuell zu beobachten war.

Bei der Buchung sollte also ein Kontrollblick auch auf die Inhalte der automatisch zugeordneten Konten geworfen werden. Außerdem ist es ratsam, überflüssige oder nicht funktionierende Importregeln zu verändern oder zu löschen. Die Importregeln können Anwender in der Buchhaltungssoftware fibu-doc unter „Datenimport“ im Unterpunkt verwalten.

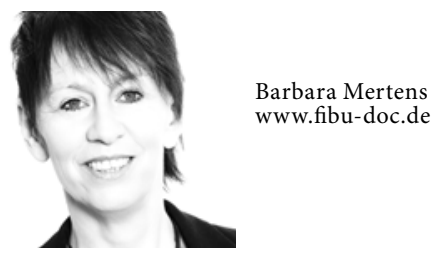

